\title{
Perioperative Outcomes of Different Surgical Methods Among Bladder Cancer Patients Undergoing Radical Cystectomy With Neobladder Urinary Diversion
}

\author{
Hak Ju Kim ${ }^{1}$, Changhee Ye ${ }^{1}$, Jin Hyuck Kim ${ }^{1}$, Hwanik Kim ${ }^{1,2}$, Sangchul Lee ${ }^{1}$, Seok-Soo Byun ${ }^{1,2}$, \\ Jong Jin $\mathrm{Oh}^{1,2}$
}

'Department of Urology, Seoul National University Bundang Hospital, Seongnam, Korea

${ }^{2}$ Deparment of Urology, Seoul National University College of Medicine, Seoul, Korea

\begin{abstract}
Purpose: To compare perioperative outcomes according to surgical methods among bladder cancer patients who underwent radical cystectomy (RC) with neobladder urinary diversion.

Materials and Methods: Between June 2007 and January 2020, 89 bladder cancer patients who received RC with neobladder urinary diversion were enrolled in this study. Patients were stratified into surgical methods - (1) open RC with neobladder (ONB) reconstruction, (2) robotassisted RC (RARC) with extracorporeal neobladder (ECNB) reconstruction, and (3) RARC with intracorporeal neobladder (ICNB) reconstruction. Perioperative outcomes were compared among the 3 groups, with major complications defined according to Clavien-Dindo grades III$\checkmark$ within 90 days. Logistic regression analysis was performed to identify significant factors for postoperative complications.

Results: Of 89 patients, 28 (31\%) had ONB, 31 (35\%) had ECNB, and 30 (34\%) had ICNB. The median operative time was 471 minutes, and the ICNB group ( 424.5 minutes) was significantly less than ONB (444.5 minutes) and ECNB groups ( 542.9 minutes) $(p=0.001)$. Transfusion rate was also significantly less in the ICNB group (13\%) $(p=0.001)$. Complications were recorded in 67 patients $(75 \%)$ and major complications in 22 of all patients $(25 \%)$. The major complication rate was significantly less in ICNB (13.4\%) than in ONB (25\%) and ECNB (35\%) $(p=0.003)$. Multivariate analysis showed surgical methods (ICNB) (odds ratio [OR], 0.709; $\mathrm{p}=0.003)$ and age (OR, 1.150; $\mathrm{p}=0.001$ ) were significant factors related to occurrence of major postoperative complications. Conclusions: RARC with ICNB reduces postoperative complications compared to ONB and ECNB.
\end{abstract}

Key Words: Urinary bladder neoplasms, Neobladder reconstruction, Postoperative complications
Received September 27, 2021

Revised October 18, 2021

Accepted October 19, 2021

\section{Corresponding author:}

Jong Jin Oh

Email: urojin@snu.ac.kr

https://orcid.org/0000-0003-0448-5992

\section{INTRODUCTION}

Radical cystectomy (RC) with extended lymphadenectomy is a standard treatment method for muscle-invasive bladder cancer (MIBC) and highrisk nonmuscle-invasive bladder cancer (NMIBC). ${ }^{1}$ Since RC became the standard approach for treatment, we have been begun performing RC, we have been trying to reduce complications such as bleeding, facilitate quicker patient recovery. Also, extremely important have been attempts to improve patient satisfaction and quality of life after surgery. As a result, the rate of robot-assisted RC (RARC) has increased in recent decades. ${ }^{2}$ RARC and

Copyright (C) The Korean Urological Oncology Society and The Korean Prostate Society. All riguts reserved. 
open RC have been shown to have no difference in oncologic outcomes. ${ }^{3-6}$ Although still somewhat controversial, ${ }^{3,7}$ recent large meta-analysis studies have shown that RARC outperforms open RC in functional outcome. ${ }^{8}$ With that in mind, we continue to try to reduce the incision size, and have tried various approaches to completing surgeries with minimal invasiveness. ${ }^{9,10}$ The current intracorporeal urinary diversion (ICUD) is the result of these efforts. In addition, there have been developments in orthotopic neobladder reconstruction which allows patients to void urine in a similar way to normal and to not require urostomy. There has been controversial for the optimal form of urinary diversion following RC. According to some recent studies, orthotopic neobladder and ileal conduit after RC show similar results in terms of global health status including some data with a better health-related quality of life in urinary outcomes. ${ }^{11-13}$ However, orthotopic neobladder reconstruction patients had significantly better physical function, and were able to pursue a more active life-style than ileal conduit patients. These patients attained urethral voiding with good continence. ${ }^{14,15}$ Several studies have demonstrated that ileal neobladder urinary diversion is a safe procedure with perioperative and long-term morbidity comparable to ileal conduit diversion. ${ }^{16}$ And several studies have also demonstrated that orthotopic neobladder reconstruction has advantages for the patient's physical function over ileal conduit diversion. ${ }^{17}$

Several studies have compared ICUD and extracorporeal urinary diversion (ECUD). ${ }^{18-20}$ To date however, most studies comparing ICUD and ECUD at RARC have been done with a combination of neobladder and ileal conduit. There are also no studies comparing ONB with ECNB and ICNB respectively. $\mathrm{RC}$ with $\mathrm{NB}$ is essentially a treatment for bladder cancer, but at the same time, it builds a new urination system for patients. Therefore, maintaining a postoperative patient's quality of life similar to that enjoyed before surgery is also a very important goal of this operation. In that sense, it is extremely important to reduce the occurrence of postoperative complications which significantly degrade quality of life after surgery. Patients with even a single complication are often subject to frequent visits to hospitals and further hospitalizations.

The goal of the study, therefore, was to find out how much the ICNB reduces patient complications, by comparing it with more invasive surgeries. We compared perioperative outcomes and complications of ONB and ECNB and ICNB for bladder cancer. Although these 3 all have the same goal, there is a difference in approach such that ONB is open-ended to all surgical procedures, ECNB is open to bowel manipulation and neobladder formations, and ICNB is robotized to all surgical procedures. That order correlates with a progression to lesser invasion. Comparing and analyzing these 3 surgical techniques for postoperative complications is a way of directly identifying the effects of minimizing invasive surgery.

\section{MATERIALS AND METHODS}

From June 2007 to January 2020, we conducted a retrospective review of 89 patients who received RC with neobladder diversion from our institute for bladder cancer. The patients involved had MIBC (T2-T4aNOM0) or high-risk NMIBC (Ta, Tis, T1). All of them had received at least 3 months of follow-up and had previously signed the informed consent. In all cases extended lymphadenectomy was performed. The extent of pelvic lymph node dissection was determined at the discretion of surgeons based on clinical preferences and judgments (based on the extent of disease, vascular disease). Males had the prostate removed. 
The data were collected for all RC with neobladder patients conducted by a single institution over a period of 14 years. ONB was performed by 3 experienced surgeons at a single institution, but 61 cases of RARC with NB were performed by one surgeon. The data included patient demographics (age, gender, body mass index), scores, marks, neoadjuvant chemotherapy, previous radiotherapy, previous abdominal surgery, hydronephrosis, surgical time (minute), estimated blood loss, and transfused units (if any), postoperative complications based on the modified Clavien-Dindo classification system, length of stay, reoperations, readmissions, interventional radiology procedures, number of resected lymph nodes, and pathologic stage.

Postoperative complications occurring during the first 90 days after the intervention were included, and these were classified as minor (Clavien-Dindo I-II) and major (Clavien-Dindo III-V). Follow-up was performed at 3, 6, and 12 months after surgery, and annually thereafter by physical examination, blood test, and abdominal computed tomography scan.

Patients were divided into 3 groups to compare and analyze preoperative clinical and pathological characteristics and postoperative complications. One-way analysis of variance was used to compare continuous variables, and chi-square test was used to compare categorical variables. Multivariable binary logistic regression was conducted to find complication predictors focused on overall complication rates in ICNB versus ECNB and ONB. All statistical analyses were performed using the IBM SPSS Statistics ver. 19.0 (IBM Co., Armonk, $\mathrm{NY}$, USA); all p-values were presented as 2-sided; p-values $<0.05$ were considered to be statistically significant.

The present study permitted by Institutional Review Board (IRB No. B-2005-613-114) of Seoul National University Bundang Hospital.

\section{RESULTS}

Table 1 shows preoperative clinical and pathological characteristics of patients undergoing open RC with neobladder and RC with ECNB and ICNB. The average age of the ONB group at 56.8 years, was lower than that of the other 2 groups $(\mathrm{p}=0.004)$ and the American Society of Anesthesiologists (ASA) physical status was also better overall than the other groups $(p=0.005)$. Preoperative hemoglobin was higher at 14.02 in the ONB group compared to the other groups $(p=0.001)$. The ECNB was 13.4 and the ICNB was 12 . There were only 3 patients receiving ONB who received neoadjuvant chemotherapy, which was less than the other groups.

Table 2 shows the perioperative outcomes and complications of patients undergoing ONB, ECNB, and ICNB. The average surgical time was significantly longer for the ECNB group at (542.9 minutes; $\mathrm{p}=0.001$ ) among the 3 groups. The average hospitalization period was 31 days in the ONB group, 20.7 days in the ECNB group, and 15.5 days in the ICNB group, significantly longer in the ONB group, and shorter in the ICNB group.

Blood transfusion rates in the 3 groups were $60 \%$ in ONB, $17 \%$ in ECNB, and $13 \%$ in ICNB, which was significantly higher in the ONB group $(p=0.001)$. There was no open conversion in ECNB and ICNB groups. At least one postoperative complication occurred in 67 patients (75\%) of all 3 groups. Postoperative complications occurred in 23 (82\%) receiving ONB, 25 (80\%) receiving ECNB, and 19 (63\%) receiving ICNB, a significantly lower incidence of complications in ICNB ( $p=0.046)$. In particular, there were 7 major complications (25\%) (Clavien-Dindo III-IV) in the ONB group and $11(35 \%)$ in ECNB group, but only 4 (13\%) in the ICNB group, a significantly lower incidence of complications in ICNB ( $\mathrm{p}=0.003)$. The most common complications were infection (38\%) 
Table 1. Preoperative clinical and pathological characteristics of patients undergoing radical cystectomy with neobladder

\begin{tabular}{|c|c|c|c|c|c|}
\hline Characteristic & Total series $(\mathrm{n}=89)$ & ONB $(n=28)$ & $\operatorname{ECNB}(n=31)$ & $\operatorname{ICNB}(n=30)$ & p-value \\
\hline Mean age (yr) & 61.48 & 56.78 & 62.25 & 65.06 & $0.004 *$ \\
\hline Sex & & & & & 0.094 \\
\hline Male & $82(92)$ & $28(100)$ & $26(84)$ & $27(90)$ & \\
\hline Female & $7(8)$ & $0(0)$ & $5(16)$ & $3(10)$ & \\
\hline Mean BMI $\left(\mathrm{kg} / \mathrm{m}^{2}\right)$ & 25.09 & 24.21 & 25.52 & 25.48 & 0.234 \\
\hline Prior abdominal and/or pelvic surgery & $18(20)$ & $7(25)$ & $8(26)$ & $4(13)$ & 0.505 \\
\hline Prior neoadjuvant chemotherapy & $24(30)$ & $3(11)$ & $12(39)$ & $17(57)$ & $0.001 *$ \\
\hline Smoking history & & & & & 0.528 \\
\hline Never smoker & $26(29)$ & $15(54)$ & $8(26)$ & $3(10)$ & \\
\hline Ever smoker & $63(71)$ & $13(46)$ & $23(61)$ & $27(90)$ & \\
\hline ASA PS classification & & & & & $0.005^{*}$ \\
\hline I & $28(31)$ & $15(54)$ & $10(32)$ & $5(17)$ & \\
\hline II & $43(48)$ & $13(46)$ & $19(61)$ & $18(60)$ & \\
\hline III & $6(67)$ & $0(0)$ & $2(7)$ & $7(23)$ & \\
\hline Preoperative hemoglobin & 13.11 & 14.02 & 13.45 & 12.03 & $0.001 *$ \\
\hline TUR pT & & & & & 0.753 \\
\hline pTx-pTa-T1-Tis & $27(30)$ & $10(36)$ & $7(23)$ & $10(33)$ & \\
\hline $\mathrm{T} 2-\mathrm{T} 4 \mathrm{a}$ & $50(56)$ & $18(64)$ & $19(61)$ & $13(43)$ & \\
\hline Pathologic stage & & & & & 0.897 \\
\hline $\mathrm{T} 2$ or less & $64(72)$ & $21(75)$ & $23(74)$ & $20(67)$ & \\
\hline $\mathrm{T} 3$ & $20(22)$ & $6(21)$ & $6(19)$ & $8(27)$ & \\
\hline $\mathrm{T} 4$ & $5(6)$ & $1(4)$ & $2(6)$ & $2(7)$ & \\
\hline Mean no. of resected nodes & 23.9 & 24.7 & 21.3 & 26.0 & 0.488 \\
\hline Positive surgical margins & $0(0)$ & $0(0)$ & $0(0)$ & $0(0)$ & \\
\hline
\end{tabular}

Values are presented as number (\%) unless otherwise indicated.

ONB: open radical cystectomy with neobladder, ECNB: extracorporeal neobladder, ICNB: intracorporeal neobladder, ASA PS: American Society of Anesthesiologists physical status, TUR: transurethral resection of bladder tumor.

$*_{p}<0.05$, statistically significant differences.

Table 2. Perioperative outcomes and complications of patients undergoing RC with NB

\begin{tabular}{|c|c|c|c|c|c|}
\hline Variable & Total series $(\mathrm{n}=89)$ & ONB $(n=28)$ & $\operatorname{ECNB}(n=31)$ & $\operatorname{ICNB}(n=30)$ & p-value \\
\hline Median operative time (min) & 471 & 444.5 & 542.9 & 424.5 & $0.001^{*}$ \\
\hline Mean hospital stay (day) & 22 & 31 & 20.7 & 15.5 & $0.001 *$ \\
\hline Hospital readmission & $34(38)$ & $12(43)$ & $16(52)$ & $6(20)$ & $0.003 *$ \\
\hline Intraoperative transfusion of $\mathrm{RBC}$ & $26(29)$ & $17(60)$ & $5(17)$ & $4(13)$ & $0.001 *$ \\
\hline Postoperative complication & $67(75)$ & $23(82)$ & $25(80)$ & $19(63)$ & $0.046^{*}$ \\
\hline Gastrointestinal & $11(12)$ & $5(18)$ & $6(19)$ & $0(0)$ & $0.041^{*}$ \\
\hline Hematological & $32(36)$ & $14(50)$ & $10(40)$ & $8(27)$ & 0.157 \\
\hline Genitourinary & $25(26)$ & $5(18)$ & $12(39)$ & $8(27)$ & 0.126 \\
\hline Cardiovascular & $1(1)$ & $1(4)$ & $0(0)$ & $0(0)$ & 0.332 \\
\hline Pulmonary & $2(2)$ & $1(4)$ & $0(0)$ & $2(7)$ & 0.578 \\
\hline Infection & $34(38)$ & $10(36)$ & $16(52)$ & $8(27)$ & 0.127 \\
\hline Wound & $14(16)$ & $7(25)$ & $5(16)$ & $2(7)$ & 0.159 \\
\hline Neurological & $1(1)$ & $0(0)$ & $0(0)$ & $1(3)$ & 0.37 \\
\hline Vascular & $0(0)$ & $0(0)$ & $0(0)$ & $0(0)$ & \\
\hline Total complications & 118 & 43 & 49 & 29 & \\
\hline Clavien-Dindo classification & & & & & $0.003 *$ \\
\hline 0 & $22(25)$ & $5(18)$ & $6(19)$ & $11(37)$ & \\
\hline $\mathrm{I}-\mathrm{II}$ & $45(51)$ & $16(57)$ & $14(45)$ & $15(50)$ & \\
\hline III-IV & $22(25)$ & $7(25)$ & $11(35)$ & $4(13)$ & \\
\hline
\end{tabular}

Values are presented as number (\%) unless otherwise indicated.

RC: radical cystectomy, NB: neobladder, ONB: open radical cystectomy with NB, ECNB: extracorporeal NB, ICNB: intracorporeal NB, RBC: red blood cell. $*_{\mathrm{p}}<0.05$, statistically significant differences. 
Table 3. Multivariable analyses of predictors of postoperative major complications (Clavien-Dindo III or IV)

\begin{tabular}{|c|c|c|c|c|}
\hline \multirow{2}{*}{ Variable } & \multicolumn{2}{|c|}{ Univariable analysis } & \multicolumn{2}{|c|}{ Multivariable analysis } \\
\hline & OR $(95 \% \mathrm{CI})$ & p-value & OR $(95 \% \mathrm{CI})$ & p-value \\
\hline Age (yr) & $1.102(1.028-1.181)$ & $0.006^{*}$ & $1.150(1.059-1.249)$ & $0.001 *$ \\
\hline Body mass index $\left(\mathrm{kg} / \mathrm{m}^{2}\right)$ & $0.998(0.858-1.162)$ & 0.983 & & \\
\hline \multicolumn{5}{|l|}{ Gender } \\
\hline Male (reference) & 1 & & & \\
\hline Female & $2.259(0.490-10.411)$ & 0.296 & & \\
\hline Prior abdominal and/or pelvic surgery & $1.434(0.428-4.803)$ & 0.559 & & \\
\hline Prior neoadjuvant chemotherapy & $0.482(0.152-1.530)$ & 0.216 & & \\
\hline \multicolumn{5}{|l|}{ OP method } \\
\hline $\mathrm{ONB}+\mathrm{ECNB}$ & 1 & & 1 & \\
\hline ICNB & $0.763(0.635-1.357)$ & $0.021 *$ & $0.709(0.416-1.061)$ & $0.003 *$ \\
\hline Intraoperative transfusion of $\mathrm{RBC}$ & $5.893(2.022-17.177)$ & $0.001 *$ & $1.014(0.301-3.420)$ & 0.982 \\
\hline Operating time & $1.002(0.998-1.007)$ & 0.335 & & \\
\hline Preoperative hemoglobin & $1.123(0.864-1.459)$ & 0.385 & & \\
\hline \multicolumn{5}{|l|}{ ASA PS classification } \\
\hline I or II (reference) & 1 & & & \\
\hline III or IV & $1.530(0.585-4.025)$ & 0.385 & & \\
\hline
\end{tabular}

OR: odds ratio, CI: confidence interval, OP: operation, ONB: open radical cystectomy with neobladder, ECNB: extracorporeal neobladder, ICNB: intracorporeal neobladder, RBC: red blood cell, ASA PS: American Society of Anesthesiologists physical status.

$*_{p}<0.05$, statistically significant differences.

and hematological complication (32\%), followed by genitourinary complication (26\%), wound problem (16\%), and gastrointestinal complication (12\%). Most of the major complications were treated by PCN inserts or double J stent inserts due to hypdronephrosis after surgery, a total of 9 patients (10\%). There were 7 cases $(8 \%)$ of reoperation under general anesthesia, and 2 of them required transurethral resection of bladder neck surgery due to urethral stricture. There were also 2 surgeries to switch back from neobladder to ileal conduit. There were 3 people who performed a wound repair under anesthesia due to a wound complication, and 3 others performed percutaneous drainage insertion with urine leakage or formula formation. No positive surgical margins were recorded among the 3 groups.

Table 3 shows multivariable analyses of predictors of postoperative complications (Clavien-Dindo III-IV). Here, we analyzed ONB and ECNB as one group, and show that ICNB had less complications than the other combined group (odds ratio, 0.709; $\mathrm{p}=0.003$ ). Covariates include age and intraoperative transfusion of red blood cell. In addition to surgical techniques, age was also associated with complications.

\section{DISCUSSION}

In this study, ICNB produced fewer major complications than ONB and ECNB. In particular, there were no significant differences in complications between ONB and ECNB except for the transfusion rate. It can be assumed that most of the complications are caused by manipulation of the bowel. In this regard, Kang's early experience of RARC with total ICUD in a paper that researched ICUD, explained that it reduces bowel exposure and has the advantage of maintaining the humidity of the bowel thus reducing the potential for fluid imbalances. ${ }^{21}$ It can be assumed that our findings also reflect that. Most of the major complications in these reports were related to urinary diversion. On the other hand, the RC part was safer and more feasible.

Our findings are also similar to those reported 
by Carrion et al. ${ }^{22}$ They reported that ICUD was associated with lower complication rates compared to ECUD. In their report, patients who underwent ECUD had a higher rate of ureteroileal strictures than those with ICUD ( $45.5 \%$ vs. $14.3 \%, \mathrm{p}=0.026$ ). Among the neobladders, ECUD developed a higher rate of urethro-neobladder stricture than ICUD (33\% vs. 0\%, p=0.044).

Conversely, Mistretta et al. ${ }^{23}$ reported that there was no statistically significant difference between ICNB versus ECNB overall (ICNB: $75.4 \%$ vs. ECNB: $72.7 \%$; $\mathrm{p}=0.9$ ), or for major complications (ICNB: $28.1 \%$ vs. ECNB: $25 \%, p=0.6$ ). Their work was most similar to ours in that they compared only in neobladder, and the number of patients was also slightly higher (ICNB: 57, ECNB: 44) than in the present study. However, in their study, the major complications rate of ICNB was $28 \%$. This was much higher than we observed. Robot surgery techniques in the study of Mistretta et al. ${ }^{23}$ may not yet have been fully mature, or the difference may be due to other limitations of the study to be described below. Our research did not just compare ECNB and ICNB, but added ONB cases, which in total led to a larger number of patients than the study of Mistretta et al. ${ }^{23}$ Our study has added significance due to the analysis and comparison of each of the following surgical approaches: ONB that are completely open, ECNB that are partially done by robots, and ICNB that are totally done by robots.

In our study, ONB was performed by several surgeons but, all of the patients who underwent RARC with NB were performed by one surgeon, and due to this, we have removed or reduced variables that would have come from differences in surgical procedure details, that could have been present if there were other surgeons.

Table 1 shows the distribution of each group was not even. If only ECNB and ICNB are analyzed and compared, there is no difference between the
2 groups, but there is a difference if the 3 groups including ONB are analyzed. In particular, the group of patients who had ONB were younger so preoperative hemoglobin was high and ASA was good. There is less neoadjuvant chemotherapy. Most ONB patients had RC before 2015, whereas most RARC patients underwent surgery after 2015. This may be part of the explanation. The criteria for patient selection and surgical indication have also changed somewhat over the past 15 years, but the average age of Koreans has risen, and the number of patients coming to our hospital has changed. In the area where our hospital is located, aging of the population has progressed more rapidly than other regions over the past decade.

The 3 surgeries showed a significant difference in operating time. Compared to other groups, the operative time is much longer in the ECNB group and the inclusion of the time to de-dock the robot in the middle may have influenced this.

The duration of the hospital stay was significantly higher with ONB. Although the recovery of ONB patients was slower, the main impact on duration is likely to be the relatively more recent emphasis on early discharge, so it is believed that this may explain the result. Currently, if complications do not occur and the recovery is successful, all RARC patients are discharged around 14 days. A similar explanation may apply as to why the readmission rate within 90 days is high in the ECNB group. The 17 patients who were readmitted in both groups required management due to complications that occurred after the operation.

In the 3 groups, $24 \%$ of patients had transfusions during the operation. Of these, $60 \%$ received ONB while $17 \%$ and $13 \%$ had ECNB and ICNB respectively. ONB clearly resulted in more bleeding during surgery.

Fewer postoperative complications occurred in the ICNB group and the difference was greater for 
major complications. The most common complications were infection (38\%) and hematological complication (32\%), followed by genitourinary complication (26\%), wound problem (16\%), and gastrointestinal complication (12\%). Since an infection focus is not always found, this complication includes patients who start on antibiotics with unknown cause of fever, and therefore may be overestimated. Hematological complication includes all cases of blood transfusion during surgery or within 90 days of surgery, so this may also have been overestimated. There were 5 gastrointestinal complications in ONB, 6 in ECNB, and none in ICNB. This finding supports the hypothesis that ICUD techniques reduce fluid loss from the bowel and minimize trauma and consequent edema to the barrier, thereby lowering gastrointestinal complications. The intervention due to hydronephrosis occurred only in the ONB and ECNB groups, and ICNB had none. Similarly, no interventions due to urethral stricture occurred in ICNB. This is consistent with the assumption that robot-assisted anastomosis of neobladder and urethra will be simplified compared to the process of externalization of the neobladder and later reinitiation of the pneumoperitoneum. As well it can be assumed that ICNB will cause less damage to tissues such as neobladder and urethra, among others.

The reported results demonstrate the potential benefits of ICNB, which is consistent with the hypothesis set in the early stages of the study. These benefits include reduced bleeding, reduced blood transfusion requirements, and shorter hospitalization. The results of the current series show that ICNB following RARC is feasible, safe, and excellent from a complications perspective. In particular, the fact that only 4 major complications occurred with the ICNB group further reinforces this hypothesis. Overall, we have confirmed once again that developing techniques that enhance minimal invasiveness is a critical part of a surgeon' $\mathrm{s}$ role.

Following are the limitations of this study. (1) The study was conducted retrospectively and nonrandomly in a single center, and the number of patients is small. And, almost RARC cases were carried out by the same surgeon. In addition, the surgeon performed all ECNB cases first, followed by all ICNB surgical cases. It was highly likely that the learning curve had an effect. Thus, some effort was made to correct the learning curve from the design stage of the study. When comparing the incidence of complications by year, the difference was not significant by year. Surgeon was enough familiar with robot handling techniques from the start, so the learning curve may not have been as large as expected. Nevertheless, the learning curve due to the difference in the timing of ECNB and ICNB will be reflected to some extent, and it is thought to be an important limitation of this study. (2) Most ONB was done much earlier compared to RARC. This difference led to differences in the distribution between each group which was uneven in the analysis of preoperative clinical characteristics of patients and the management of patients. As a result, not only surgical techniques but also postoperative patient care techniques have varied over time, which may have affected the results of this analysis. However, the neobladder formation techniques on ONB and ECNB were the same. This makes this study more convincing. And in terms of the preoperative condition of patients, the ONB group had the best patients, followed by ECNB and ICNB were worst. The differences in postoperative complications shown in this study are likely to be underestimated. (3) The advantages of the enhanced recovery after-surgical (ERAS) method have been known for years, ${ }^{24-26}$ and as a result, most ONB patients have not been ERAS- 
applied, while most ICNB patients have been. It is, therefore, possible that this affected some of the outcomes. (4) The difference in the timing of the surgery also makes the difference in neoadjuvant chemotherapy rate between each group. Almost ONB surgery was performed in the past when neoadjuvant chemotherapy was not performed. It also seems to be an important limitation. (5) If the postoperative complications were divided into early and postmerger complications, another meaningful interpretation may have been found. Nevertheless, our work provides valuable data as the first comparison of ONB, ECNB, and ICNB. Studies will be needed in future which avoids the above limitations while adding oncologic outcomes.

\section{CONCLUSIONS}

Compared to other neobladder reconstruction methods, RARC with intracorporeal neobladder reconstruction reduces postoperative complications such as postoperative ileus, uretero-neobladder, and urethra-neobladder stricture during the follow-up period. It also reduces the duration of hospitalization after surgery and the rate of readmission after discharge. Overall, ICNB improves postoperative patient satisfaction.

\section{CONFLICT OF INTEREST}

The authors claim no conflicts of interest.

\section{ORCID}

Hak Ju Kim, https://orcid.org/0000-0002-9960-698X Changhee Ye, https://orcid.org/0000-0002-2328-8436 Jin Hyuck Kim, https://orcid.org/0000-0002-8702-7008 Hwanik Kim, https://orcid.org/0000-0001-5201-2490 Sangchul Lee, https://orcid.org/0000-0003-0844-6843 Seok-Soo Byun, https://orcid.org/0000-0001-9356-9500
Jong Jin Oh, https://orcid.org/0000-0003-0448-5992

\section{REFERENCES}

1. Witjes JA, Lebret T, Compérat EM, Cowan NC, De Santis M, Bruins HM, et al. Updated 2016 EAU guidelines on muscle-invasive and metastatic bladder cancer. Eur Urol 2017;71:462-75.

2. Gill IS, Cacciamani GE. The changing face of urologic oncologic surgery from 2000-2018 (63 141 patients)-impact of robotics. Eur Urol Suppl 2019; 18:e656-7.

3. Parekh DJ, Reis IM, Castle EP, Gonzalgo ML, Woods ME, Svatek RS, et al. Robot-assisted radical cystectomy versus open radical cystectomy in patients with bladder cancer (RAZOR): an open-label, randomised, phase 3, non-inferiority trial. Lancet 2018; 391:2525-36.

4. Khan MS, Omar K, Ahmed K, Gan C, Van Hemelrijck M, Nair R, et al. Long-term oncological outcomes from an early phase randomised controlled three-arm trial of open, robotic, and laparoscopic radical cystectomy (CORAL). Eur Urol 2020;77:1108.

5. Elsayed AS, Gibson S, Jing Z, Wijburg C, Wagner AA, Mottrie A, et al. Rates and patterns of recurrences and survival outcomes after robot-assisted radical cystectomy: results from the International Robotic Cystectomy Consortium. J Urol 2021;205:407-13.

6. Wilson TG, Guru K, Rosen RC, Wiklund P, Annerstedt $\mathrm{M}$, Bochner $\mathrm{BH}$, et al. Best practices in robotassisted radical cystectomy and urinary reconstruction: recommendations of the Pasadena Consensus Panel. Eur Urol 2015;67:363-75.

7. Satkunasivam R, Tallman CT, Taylor JM, Miles BJ, Klaassen Z, Wallis CJ. Robot-assisted radical cystectomy versus open radical cystectomy: a metaanalysis of oncologic, perioperative, and complication-related outcomes. Eur Urol Oncol 2019;2:4437.

8. Clement KD, Pearce E, Gabr AH, Rai BP, Al-Ansari 
A, Aboumarzouk OM. Perioperative outcomes and safety of robotic vs open cystectomy: a systematic review and meta-analysis of 12,640 cases. World J Urol 2021;39:1733-46.

9. Wilson TG, Guru K, Rosen RC, Wiklund P, Annerstedt $\mathrm{M}$, Bochner $\mathrm{BH}$, et al. Best practices in robotassisted radical cystectomy and urinary reconstruction: recommendations of the Pasadena Consensus Panel. Eur Urol 2015;67:363-75.

10. Hemal AK. Role of robot-assisted surgery for bladder cancer. Curr Opin Urol 2009;19:69-75.

11. Goldberg H, Baniel J, Mano R, Rotlevy G, Kedar D, Yossepowitch O. Orthotopic neobladder vs. ileal conduit urinary diversion: a long-term quality-oflife comparison. Urol Oncol 2016;34:121.e1-7.

12. Ziouziou I, Irani J, Wei JT, Karmouni T, El Khader $\mathrm{K}$, Koutani A, et al. Ileal conduit vs orthotopic neobladder: which one offers the best health-related quality of life in patients undergoing radical cystectomy? A systematic review of literature and meta-analysis. Prog Urol 2018;28:241-50.

13. Cerruto MA, D’Elia C, Siracusano S, Saleh O, Gacci M, Cacciamani G, et al. Health-related quality of life after radical cystectomy: a cross-sectional study with matched-pair analysis on ileal conduit vs ileal orthotopic neobladder diversion. Urology 2017;108:82-9.

14. Philip J, Manikandan R, Venugopal S, Desouza J, Javlé PM. Orthotopic neobladder versus ileal conduit urinary diversion after cystectomy-a qualityof-life based comparison. Ann R Coll Surg Engl 2009;91:565-9.

15. Nam JK, Lee DH, Park SW, Chung MK. Long-term functional outcomes of ileal neobladder reconstruction following radical cystectomy. Korean J Urol Oncol 2017;15:21-7.

16. Gburek BM, Lieber MM, Blute ML. Comparison of Studer ileal neobladder and ileal conduit urinary diversion with respect to perioperative outcome and late complications. J Urol 1998;160(3 Part 1): 721-3.
17. Dutta SC, Chang SS, Coffey CS, Smith JA, Jack G, Cookson MS. Health related quality of life assessment after radical cystectomy: comparison of ileal conduit with continent orthotopic neobladder. J Urol 2002;168:164-7.

18. Hussein AA, May PR, Jing Z, Ahmed YE, Wijburg CJ, Canda AE, et al. Outcomes of intracorporeal urinary diversion after robot-assisted radical cystectomy: results from the International Robotic Cystectomy Consortium. J Urol 2018;199:1302-11.

19. Lenfant L, Verhoest G, Campi R, Parra J, Graffeille V, Masson-Lecomte A, et al. Perioperative outcomes and complications of intracorporeal vs extracorporeal urinary diversion after robot-assisted radical cystectomy for bladder cancer: a real-life, multiinstitutional french study. World J Urol 2018;36: 1711-8.

20. Zhang JH, Ericson KJ, Thomas LJ, Knorr J, Khanna A, Crane A, et al. Large single institution comparison of perioperative outcomes and complications of open radical cystectomy, intracorporeal robotassisted radical cystectomy and robotic extracorporeal approach. J Urol 2020;203:512-21.

21. Kang SG, Ko YH, Jang HA, Kim J, Kim SH, Cheon $\mathrm{J}$, et al. Initial experience of robot-assisted radical cystectomy with total intracorporeal urinary diversion: comparison with extracorporeal method. J Laparoendosc Adv Surg Tech A 2012;22:456-62.

22. Carrion A, Piñero A, Raventós C, Lozano F, Díaz F, Morote J. Comparison of perioperative outcomes and complications of robot assisted radical cystectomy with extracorporeal vs intracorporeal urinary diversion. Actas Urol Esp (Engl Ed) 2019;43:277-83.

23. Mistretta FA, Musi G, Collà Ruvolo C, Conti A, Luzzago S, Catellani M, et al. Robot-assisted radical cystectomy for nonmetastatic urothelial carcinoma of urinary bladder: a comparison between intracorporeal versus extracorporeal orthotopic ileal neobladder. J Endourol 2021;35:151-8.

24. Persson B, Carringer M, Andrén O, Andersson SO, Carlsson J, Ljungqvist O. Initial experiences with 
the enhanced recovery after surgery (ERAS $®$ ) protocol in open radical cystectomy. Scand J Urol 2015;49:302-7.

25. Pang KH, Groves R, Venugopal S, Noon AP, Catto JW. Prospective implementation of enhanced recovery after surgery protocols to radical cystec- tomy. Eur Urol 2018;73:363-71.

26. Kukreja JB, Shi Q, Chang CM, Seif MA, Sterling BM, Chen TY, et al. Patient-reported outcomes are associated with enhanced recovery status in patients with bladder cancer undergoing radical cystectomy. Surg Innov 2018;25:242-50. 